\title{
Validation of the Wistar-kyoto Rat Kept in Solitary Housing as an Animal Model for Endogenous Depression Using Voxel-based Morphometry
}

Takanobu Yoshii ( $\square$ takanon@koto.kpu-m.ac.jp )

Kyoto Prefectural University of Medicine

Naoya Oishi

Kyoto University

Yasutaka Sotozono

Kyoto Prefectural University of Medicine

Anri Watanabe

Kyoto Prefectural University of Medicine

Yuki Sakai

Advanced Telecommunications Research Institute International

Shunji Yamada

Kyoto Prefectural University of Medicine

Kenichi Matsuda

Kyoto Prefectural University of Medicine

Masamitsu Kido

Kyoto Prefectural University of Medicine

Kazuya Ikoma

Kyoto Prefectural University of Medicine

Masaki Tanaka

Kyoto Prefectural University of Medicine

Jin Narumoto

Kyoto Prefectural University of Medicine

\section{Research Article}

Keywords: Major depressive disorder, Wistar-Kyoto (WKY), solitary housing, animal model, endogenous depression, voxel-based morphometry

Posted Date: June 17th, 2021

DOI: https://doi.org/10.21203/rs.3.rs-612118/v1 
License: (c) (i) This work is licensed under a Creative Commons Attribution 4.0 International License. Read Full License 


\section{Abstract}

Major depressive disorder is a common psychiatric condition that is often resistant to medication. The Wistar-Kyoto (WKY) rat has been suggested as an animal model of endogenous depression; however, it is challenging to translate results obtained in animal models into humans. Solitary housing is a mild stress paradigm that could simulate the environment of depressive patients with limited social activity due to symptoms. We used voxel-based morphometry to directly compare the solitary-housed WKY rat model with data from previous human studies, and validated our results with behavioural studies and correlation analyses. Atrophy in WKY rats was detected in the ventral hippocampus, caudate putamen, lateral septum, cerebellar vermis, and cerebellar nuclei ( $p<0.05$, corrected for family-wise error rate). Further, locomotor behaviour was negatively correlated with hippocampal atrophy and positively correlated with atrophy of the cerebellar vermis. The regions of brain atrophy validate WKY rats as an animal model for endogenous depression and can aid the translation of study results to humans. Our study also reveals the possibility of a cerebellar contribution to depression.

\section{Introduction}

Major depressive disorder (MDD) is one of the most common psychiatric disorders. Globally, more than 264 million people of all ages suffer from depression ${ }^{1}$, and a meta-analysis of studies conducted in 30 countries from 1994 to 2014 reported that the aggregate prevalence of depression was $12.9 \%^{2}$. However, more than $60 \%$ of patients cannot achieve sustained remission with any single antidepressant drug therapy, and one-third of patients receive a diagnosis of treatment-resistant MDD ${ }^{3}$.

Voxel-based morphometry (VBM) is a well-established comprehensive analysis for structural brain magnetic resonance imaging (MRI) ${ }^{4,5}$. In human clinical studies, meta-analyses have already investigated common variations in $\mathrm{MDD}^{6}$, but the reported regions with significant atrophy have been gradually shifting from the frontal regions, including the anterior cingulate cortex $(A C C)^{7,8}$, to parietal and limbic regions $^{9,10,11}$ and the cerebellum ${ }^{12}$. Further, many people with bipolar disorder (BD) are misdiagnosed with $\mathrm{MDD}^{13,14}$, and it is possible that reduced responsiveness to antidepressants may be due to veiled bipolar mood variations. A recent meta-analysis comparing MDD, BD, and healthy controls found atrophy in the dorsolateral prefrontal cortex, hippocampus, and cerebellum in patients with MDD ${ }^{15}$. However, additional studies investigating these changes are still needed, and animal experiments may be the most suitable method for doing so.

The Wistar-Kyoto (WKY) rat was initially established from Wistar rats as a normotensive control strain for spontaneously hypertensive rats ${ }^{16}$. The WKY rat has since been used to assess a wide range of behavioural changes linked to symptoms seen in human MDD, including exaggerated stress responses, increased anhedonia, learned helplessness, novelty-induced hypophagia, neophobia, decreased locomotor activity, and increased anxiety, allowing for face validity as an endogenous depression model ${ }^{17-20}$. Endocrinological and neurophysiological analysis has been conducted for construct 
validation, and increased basal corticosterone levels, Adrenocorticotropic hormone (ACTH) levels ${ }^{21,22}$, and basal noradrenaline neurons activity ${ }^{23}$ were reported. Reduced Brain-derived neurotrophic factor (BDNF) levels in the frontal cortex and hippocampus ${ }^{24}$, as well as reduced serum BDNF response to mild stress ${ }^{25}$ have also been reported in WKY rats. Alterations in serotonergic and dopaminergic system markers have also been reported, which is similar to changes observed in MDD patients ${ }^{26}$. Further, WKY rats are considered a model of treatment-resistant depression because they are hyporesponsive to antidepressants ${ }^{16,26,27}$, and although predictive validities are still in discussion, this is important because of the similar response often observed in MDD patients.

Many depressed patients exhibit restricted social activity ${ }^{28}$, and there are significant associations between reduced social skills and depression ${ }^{29}$. We therefore suggest that an isolation paradigm in animal studies could mimic the typical environment of depressive patients. WKY rats within an isolation paradigm often exhibit enhanced depressive symptoms ${ }^{30-34}$.

The mechanisms underlying treatment resistance in MDD patients are unclear. Although several clinical studies have investigated this, the involvement of drug-naive patients presents challenges because the concepts of treatment resistance and drug naivety are incompatible. Conducting VBM in an animal model may be the first step in connecting the findings of animal and human studies.

We aim to validate the similarities in atrophic brain regions between WKY rats and humans with MDD with an animal VBM study using MRI and comparing results with clinical MDD studies. We also examined the correlations between behavioural and morphological changes to validate WKY rats as a model for depression.

\section{Results}

\section{Two open-field tests (OFTs)}

Behavioural changes were measured in two OFT trials. Strains $\times$ trials $\times$ parameters $(F(1,34)=3.308, p<$ $0.001)$, strains $\times$ parameters $(F=187.4, p<0.001)$, and parameters $(F=11.424, p<0.001)$ exhibited significant interactions; however, the interactions of strains $\times$ trials $(F=3.663, p=0.068)$, parameters $\times$ trials $(F=0.391, p=0.999)$, and trials $(F=0.365, p=0.552)$ were not significant. In addition, a significant difference was observed in body weight (Wistar: $350.24 \pm 9.48 \mathrm{~g}$; WKY: $241.37 \pm 9.523 \mathrm{~g} ; t=11.06$; $p<$ 0.001 ) and TIV, which was calculated using the MRI data (Wistar: $2166.46 \pm 153.40 \mathrm{~mm}^{3}$; WKY: 2056.32 $\pm 97.34 ; t=2.19 ; p=0.041$ ). All locomotion parameters were significantly reduced in WKY rats in both OFT trials (Fig. 1b-h) and significant differences in anxiety behaviour were observed after isolation (second trial). In WKY rats, wall-side time was significantly higher (Fig. 1i) and the proportion of time and total time in the centre region were significantly lower in the second trial (Fig. 1j-k). See Supplementary Table 1 for the statistical results of strain comparisons. 
When comparing the two trials, we found that many locomotive parameters significantly decreased, including the total distance, total movement duration, total number of movement episodes, duration per movement, and average speed (Fig. 1b-h). Wall-side time increased in WKY rats in the second trial (Fig. 1i), and only moving speed significantly increased in Wistar rats. See Supplementary Table 2 for statistical results of the trial comparisons. Together, this indicates that the escape activity of WKY rats was disrupted from the initial phase and decreased more after an additional seven days in individual housing. These rats also showed enhanced anxiety parameters after the seven-day isolation period.

When comparing the time spent in each block between the two trials, we found that in the second trial, Wistar rats stayed significantly longer in block 25 and spent less time in block 3 (Fig. 1I), whereas WKY rats stayed significantly longer in block 1 and spent less time in block 6 (Supplementary Table 2;

Fig. $1 \mathrm{~m}$ ). When comparing strains, Wistar rats stayed longer in distant blocks and the centre area in both trials (Supplementary Table 1; Supplementary Fig. 1b and 1c). These results demonstrate that WKY rats did not flee to distant blocks, and represents the despair of an endogenous depression model.

\section{Freezing time and low locomotion during forced swim test (FST)}

Freezing time was compared in both strains during the 6-min FST and was assessed as a measure of disrupted locomotion, a depressive trait. Freezing time, or the percent of time immobile, was significantly longer in WKY rats than Wistar rats (Wistar: 1.77\% $\pm 0.59 \%$; WKY: $24.45 \% \pm 5.25 \% ; t=-4.297 ; p<0.01$; Fig. 1n). Total movement distance, a measure of locomotor activity, was also significantly less in WKY rats than Wistar rats (Wistar: $1211.84 \pm 46.42 \mathrm{~cm}$; WKY: $889.55 \pm 56.56 \mathrm{~cm} ; t=4.404 ; p<0.01$; Fig. 10). WKY rats thus exhibited reduced locomotor activity and more immobility compared to Wistar rats during the initial phase of this study. The data from two Wistar rats were excluded due to camera failure (Wistar: $n=11$, WKY: $n=13$ ).

\section{Results of voxel-based morphometry (VBM) and voxel- based correlation analysis}

VBM was performed after MRI acquisition. The VBM results from axial slices are presented in Fig. 2a and 2b. No significant hypertrophic clusters were detected (height level, $p<0.001$; cluster family-wise error (FWE) corrected, $p<0.05$ ), although there were seven significant atrophic clusters (clusters A-G; Fig. 2a; Table 1): the cerebellum (cluster A); the bilateral ventral hippocampus, extending to the septum (cluster B); the bilateral basolateral amygdala (clusters $\mathrm{C}$ and $\mathrm{E}$ ); the left secondary somatosensory cortex, extending to the insular cortex (cluster D); and the pituitary gland (cluster $\mathrm{G}$ ). With a stricter threshold ( $p<$ 0.05; FWE-corrected; minimum voxel size: 19), the following clusters remained (Fig. 2b; Table 2): the cerebellar nuclei (clusters A1, A3, A4, and A5), the rostral cerebellar vermis (cluster A2), the caudal cerebellar vermis (cluster F1), the left ventral hippocampus (cluster B1), the right caudate putamen (cluster B2), and the right lateral septum (cluster B3). 
We also performed a voxel-based correlation analysis between the parameters from the second OFT, except for time spent in each block and grey matter concentration, in WKY rats. We observed clusters in different brain areas with negative correlations between grey matter volume and certain behaviours typically studied in depression research. We observed negative correlations between total movement duration and total number of movement episodes in right habenula clusters (Fig. 3a and 3b); between distance per movement and clusters in the left amygdala (Fig. 3c); total number of movement episodes and clusters in the left ventral hippocampus (Fig. 3b); and movement speed and clusters in the right lateral septum (Fig. 3d). On the other hand, clusters with positive correlations were detected in the cerebellar vermis and pituitary gland: a cluster in lobule 9 in the caudal vermis was positively correlated with distance per movement (Fig. 4a), duration per movement (Fig. 4b), and moving speed (Fig. 4c); a cluster in lobule 3 in the rostral vermis correlated with duration per movement (Fig. 4a); and a cluster in the pituitary gland correlated with duration per movement (Fig. 4b). However, no significant cluster was found to correlate grey matter with wall-side time, percent of time in the centre region, or total time in the centre region.

\section{Discussion}

VBM revealed significant atrophy in the following brain areas of WKY rats: the left ventral hippocampus, the right caudate putamen, the right lateral septum, the cerebellar vermis, and the cerebellar nuclei. According to a recent clinical meta-analysis ${ }^{15}$, the VBM results comparing WKY and Wistar rats are similar to those in human clinical MDD studies, although the largest atrophic cluster was observed in the cerebellum. The results of the two OFT trials and FST indicates the behavioural characteristics of WKY rats as a depression model, namely locomotion disruption and reduced escape activity. Voxel-based correlation analysis revealed areas correlated with escape behaviour in the second OFT. We observed negative correlations in the hippocampus, which agree with the results of a previous animal study 35 , and the right habenula, as well as a positive correlation in the cerebellar vermis. Our VBM results indicate similar anatomical changes in WKY rats as in humans, validating their use as an endogenous depression model.

Surprisingly, the most significant atrophic cluster was detected in the cerebellum with the moderate threshold. The clusters observed in the cerebellar nuclei and vermis in the 3rd and 9th lobules remained after the stricter threshold was applied. A recent meta-analysis reported an atrophic cluster in the cerebellum of MDD patients ${ }^{12}$, and other studies have suggested cerebellar involvement in modulating various aspects of mood and cognition ${ }^{36-38}$. Hypertrophy has been observed in the cerebellum of depressed patients after successful treatment with electroconvulsive therapy ${ }^{39}$ as well as in the cerebellar vermis after the use of chronic antidepressant medication ${ }^{40}$. Another recent meta-analysis detected a hypertrophic cluster in the cerebellar vermis in BD patients relative to MDD patients ${ }^{15}$. The cerebellar atrophy in WKY rats thus reflects MDD but not BD. In addition, positive correlations between atrophic clusters and certain behaviours were detected in the vermis (9th lobule), although we could not confirm positive correlations with other atrophic brain regions, such as the hippocampus. The cerebellar vermis is 
included in the cerebrocerebellum and receives target projections from motor areas ${ }^{41}$. According to our OFT results, atrophy in the vermis may lead to disrupted escape activity. Cerebellar atrophy may trigger behavioural disturbances and induce an inability to perform even in the presence of motivation. The role of the cerebellum in emotional processing ${ }^{42}$ is still being discussed, and the contribution of the cerebellum to depressive symptoms should be investigated further.

We observed a significant cluster extending from the hippocampus to the septal region, somewhat including the thalamus, with the moderate threshold, and a cluster in the left ventral hippocampus remained after the stricter threshold was applied. This demonstrates significant atrophy in the ventral hippocampus and an atrophic trend in the dorsal hippocampus (Supplementary Fig. 3) A previous animal study reported negative correlations between locomotion and grey matter concentration in the ventral hippocampus ${ }^{35}$. Because the hippocampus is a common region of interest in MDD research, as supported by a recent meta-analysis ${ }^{15}$, the atrophy observed in the hippocampus and parahippocampal regions in our model indicates similarity to human studies. However, WKY rats have been reported to be vulnerable to stress ${ }^{16}$, and differential roles of the ventral (stress, emotion) and dorsal (cognition) hippocampi have been discussed ${ }^{43,44}$, so the atrophy we observed might be stress-related.

Our data demonstrates a negative correlation between escape behaviour and the right habenula as well as volume reduction. The relationship between the volume of the habenula and its effect on escape activity should be investigated further. It has been proposed that the lateral habenula could systematically learn to expect an adverse outcome, and frequent neural firing may lead to a state of continuous disappointment and hopelessness ${ }^{45}$. A significant volume reduction in the habenula of depressive patients has also been reported ${ }^{46}$, and the habenula is considered a critical region in MDD research ${ }^{47}$. Ketamine-induced metabolic reduction in the right habenula of treatment-resistant depression patients has been reported ${ }^{48}$, and the habenula may be a potential target for future research on treatment-resistant depression.

Brain atrophy observed in the right lateral septum neighbouring the fimbria remained when the stricter threshold was applied, and a negative correlation with escape behaviour was detected. Although the septal area is rarely described in clinical depression studies using MRI, previous animal studies reported that the septal nuclei are involved in stress responses ${ }^{49}$. In addition, lesion studies of the septum reported aggressive behaviours ${ }^{50,51}$. Patients with clinical MDD exhibit symptoms of ambivalence, lost motivation, reduced locomotor activity, and increased impulsivity and irritation. Further study is warranted to clarify whether the atrophy that extends from the ventral hippocampus to the septum contributes to the complex symptoms observed in MDD.

Only two clusters including the amygdala were detected using the moderate threshold, although a negative correlation to movement duration was detected in the left amygdala. Some volumetric studies in MDD patients have reported increased amygdala volumes ${ }^{52,53}$, whereas others have reported reduced volumes $^{54,55}$. A meta-regression analysis in first-episode MDD patients showed negative correlations 
between the volume of the right amygdala and Hamilton Depression Rating Scale score ${ }^{10}$. In another recent study, patients with treatment-resistant MDD exhibited blunted amygdala activity during a facial recognition task ${ }^{56}$. Further research is thus warranted to determine the relationship between amygdala volume and treatment-resistant MDD.

Our results indicated atrophy from the secondary somatosensory cortex extending to the insular cortex, although this did not remain after the stricter threshold was applied. Therefore, we cannot determine any major contribution of this site in MDD. However, a human study using VBM analysis ${ }^{57}$ and some metaanalyses $7,10,11$ have reported insular cortex atrophy in MDD patients, and our VBM data seems to be consistent with these studies. Our voxel-based correlation analysis could not detect a significant cluster in the somatosensory or insular cortices, and no correlation between these areas and behaviour could be confirmed in WKY rats. Insular cortex atrophy is commonly observed in many psychiatric disorders ${ }^{58}$, so analysis of this region in post-mortem brain studies may help to elucidate genetic spectra across various psychiatric disorders ${ }^{59}$, including MDD.

To the best of our knowledge, a reduction in pituitary gland volume has not previously been reported in WKY rats. However, it has been demonstrated that isolation paradigms elevate plasma ACTH levels without changes in corticosterone levels ${ }^{30}$, and we cannot rule out the possibility that isolation exhausted pituitary ACTH in WKY rats, resulting in atrophy. However, our study showed that pituitary atrophy was positively correlated with locomotion behaviour, so future studies are required to determine whether this atrophy is a biomarker of treatment resistance.

In this study, we were unable to confirm any ACC atrophy in WKY rats. Although atrophy in the frontal regions containing the $A C C$ has been reported in MDD patients ${ }^{7,60}$, a medication wash-out MDD study reported an increase in ACC volume ${ }^{9}$. Miniscule changes have been observed in medial prefrontal regions in WKY rats ${ }^{16}$, and we cannot completely rule out small anatomical variations corresponding with strain differences. In addition, deep brain stimulation of the prefrontal region in WKY rats has been shown to rescue disrupted locomotor activity ${ }^{61}$. The contribution of frontal brain atrophy to depression symptoms remains unclear.

Both the OFT and FST were used to validate depressive behaviour. Although we did not find any literature showing the impact of a 10 min OFT on brain morphology, we cannot rule out the carry-over effect of the OFT upon the FST in this experimental paradigm. OFT has been criticized as an anxiety-detecting measure $^{62}$, and it has been discussed that immobility during the FST is not indicative of despair, but a coping benefit from learning and memory to promote behavioural adaptation and survival ${ }^{63}$. We cannot rule this possibility out when interpreting the enhanced preference for passive survival coping strategies in WKY rats; however, our data simultaneously demonstrated low locomotor activity during both the FST and OFT. Disruption of exploratory locomotion during the OFT, where candidates could exhibit coping behaviours to escape from isolation more easily than in the FST, would not relate to survival. Although Wistar rats exhibited prolonged stays in distant blocks, WKY rats did not, although moderate stress is 
expected to induce this coping behaviour. Our behavioural results thus show reduced escape behaviour both in neutral and threatening situations, which seems to represent the presence of despair. We assume that these results indicate face validity for this model of endogenous depression.

In this study, we housed rats individually, and cannot completely rule out the effect of isolation on brain atrophy. However, there are few reports on microscopic morphological changes induced by social isolation (besides maternal separation) within the first two weeks; only one report describes olfactory bulb atrophy induced by isolation ${ }^{64}$. Although it has been proposed that a social isolation paradigm might have adverse effects for recovery interventions, such inducing neurogenesis in the hippocampus following exercise $e^{65,66}$, no significant volumetric changes in the hippocampus were reported during two weeks of social isolation using the region of interest-volumetric MRI method ${ }^{64}$. In another endogenous depression model, the Flinders sensitive line, five weeks of isolation induced erasure of depression-like behaviour ${ }^{67}$. Whether isolation can be dismissed as stress in animal endogenous depression models is still an interesting question. We suspect that the effect of isolation stress in the first two weeks might be too small to affect brain morphology in animal depression models, although we cannot rule out the possibility that genetic differences might enhance environmental effects.

We used an ex vivo MRI protocol to achieve the quality necessary for VBM. However, ex vivo MRI measurements have been discussed as less preferable for structural brain studies due to potential displacement, disrupted brain tissue integrity, and deformations resulting in artifacts ${ }^{68}$. This method may cause a global shrinkage in brain volume $(\sim 10 \%)$ and damage is most notable in the cerebellum, olfactory bulb, and cortex ${ }^{69,70}$. We therefore cannot completely rule out any data noise caused by the fixation method used. Spatially-normalized images in VBM analysis should have a relatively high resolution in humans, and grey matter segmentation is not excessively confounded by partial volume effects ${ }^{4}$, but these assumptions may not hold in small animals. For in vivo MRI in small animals, it is difficult to prevent motion artifacts and there are resolution disadvantages. In particular, it has been reported that the thalamus and hypothalamus can be more properly segmented using ex vivo MRI data ${ }^{69}$. There is also an MRI study reporting that the volume of the midbrain, hippocampus, thalamus, and cortex were relatively unaffected by perfuse-fixation ${ }^{70}$. The significant data observed in parahippocampal regions and cerebellar nuclei in this study might have benefitted from using ex vivo MRI techniques.

This study shows that single-housed WKY rats, as an endogenous depression model, demonstrated morphological similarities to MDD in humans. Correlations were determined to exist between the habenula and cerebellar vermis with inhibited escape behaviour via voxel-based correlation analysis. Although the contributions of the cerebellum ${ }^{36}$ and habenula ${ }^{47}$ to MDD have been discussed, there is insufficient research and therapies directly targeting these areas. We believe that WKY rats would be a good model for exploring the mechanisms of MDD and address therapeutic challenges.

\section{Materials And Methods}




\section{Animals}

Eight-week-old Wistar $(n=13)$ and WKY rats $(n=13)$ were obtained from Charles River Laboratories Japan. (Yokohama, Japan). Initially, all rats were housed individually, maintained in all air-conditioned rooms $\left(23^{\circ} \mathrm{C} \pm 1^{\circ} \mathrm{C}\right)$ on a $12 \mathrm{~h}$ light/dark cycle (6:00-18:00 light), and fed food and water ad libitum. All experiments were approved by the Committee on Animal Research at Kyoto Prefectural University of Medicine and were conducted according to the animal care guidelines of the National Institutes of Health and ARRIVE guidelines ${ }^{71}$. We minimised the number of animals used and their suffering.

\section{Behavioural measurements and analysis}

Two exposures to an open field were done to compare the anxiety-related and locomotive behaviour of the two rat strains. The first exposure trial provided a novel environment, while the second trial allowed us to observe exploratory motivation after the rats were kept in individual cages. The first exposures and behavioural measurements were conducted three days after rats were delivered from the distributer. Rats were then subjected to a 6-min forced swim test (FST). After seven additional days of being housed individually, the second open-field test (OFT) trial was performed (Fig. 1a).

\section{Open field test (OFT)}

Animals were placed in an OFT apparatus $\left(90 \times 90 \times 40 \mathrm{~cm}^{3}\right)$ to freely explore the field for $10 \mathrm{~min}$, and an overhead camera and tracking software recorded all movements (O'Hara and Co., Tokyo, Japan). The surface and walls of the apparatus were cleaned with ethanol before and after each session. To track movements, the apparatus was divided into 25 blocks and blocks 7 to 9, 12 to 14 , and 17 to 19 were defined as the centre region (Supplementary Fig. 1a). The total distance $(\mathrm{cm})$, total movement duration (s), total number of movement episodes, average speed $(\mathrm{cm} / \mathrm{s})$, moving speed $(\mathrm{cm} / \mathrm{s})$, distance per movement $(\mathrm{cm})$, duration per movement (s), wall-side time (s), percent time in centre region (\%), total time in centre region (s), and the time spent in each of the 25 blocks (s) were measured as behavioural parameters (hereafter simply referred to as parameters). Statistical analysis was performed with a repeated-measures general linear model (GLM) with a covariate of total intracranial volume (TIV) and a modified multiple comparisons test using Bonferroni's method and SPSS Statistics ver. 23.0.0.2 (IBM, Armonk, NY, USA).

\section{Forced swim test (FST)}

After the first OFT measurements, animals were placed in a $60 \mathrm{~cm} \times 20 \mathrm{~cm}$ acrylic resin cylinder (O'Hara and Co.) containing water (height: $30 \mathrm{~cm}$; temperature: $23^{\circ} \mathrm{C}$ ) for $6 \mathrm{~min}$. The time spent immobile, which included floating and movements necessary for breathing, was assessed using video tracking software with two cameras (O'Hara and Co.). The total duration of swim movements was also measured. The 
freezing time was defined as the time during which the two cameras simultaneously recorded no movement. Two-tailed two-sample $t$-tests (Welch's method) were applied for the statistical analysis.

\section{Tissue preparation}

After the behavioural measurements were taken, Wistar and WKY rats were deeply anesthetised with pentobarbital, then perfused and fixed with $4 \%$ paraformaldehyde. Rat skulls, including the brain, were removed and immersed in the same fixative overnight, then stored at $4^{\circ} \mathrm{C}$ until MRI.

\section{Ex vivo brain MRI}

We modified our previously established ex vivo brain MRI technique ${ }^{72}$ to acquire images of four rats simultaneously using a cradle (Rehabitech, Kyoto, Japan; Supplementary Fig. 2). Briefly, a Helmholtz small-volume coil (probe dimension: 108/63) was used for both radiofrequency excitation and signal detection in a 7T Varian system (Agilent Technologies, Palo Alto, CA, USA). Fast spin-echo 3D volume was collected (TR: $2000 \mathrm{~ms}$; TE: $20 \mathrm{~ms}$; flip angle: $30^{\circ}$; FOV: $90 \times 40 \times 40 \mathrm{~mm}^{3}$ ) and a bias field inhomogeneity correction was applied to the acquired scans (acquisition matrix: $256 \times 128 \times 128$; zero filled to: $512 \times 256 \times 256$; final voxel resolution: $0.176 \times 0.156 \times 0.156 \mathrm{~mm}^{3}$ ).

\section{Voxel-based morphometry (VBM) and voxel-based correlation analysis}

MRI images were segmented to identify grey matter and spatially normalised with diffeomorphic anatomical registration using the exponentiated lie algebra (DARTEL) protocol in statistical parametric mapping software ver.12 (SPM12) and the in-house rat brain template and probabilistic maps created in our previous study ${ }^{72}$. We also followed the VBM methods detailed previously ${ }^{72}$. Briefly, experimental brain MR images were denoised with a 3D non-local means filter, co-registered, and segmented to identify grey matter before spatial normalisation ${ }^{72}$. Jacobian determinants from the DARTEL procedure were calculated and used for modifying the processed grey matter images. The images were resampled into $0.15 \times 0.15 \times 0.15 \mathrm{~mm}^{3}$ voxels and smoothed with an isotropic Gaussian kernel of $0.8 \mathrm{~mm}$ in the original space, which was full-width at half-maximum. For the group-level statistical analysis, we performed voxel-by-voxel two-sample $t$-tests across the whole brain by applying the GLM. TIV was set as a covariate in all analyses. The significance level was first set at a cluster height threshold of $p<0.001$, with a determinant extent threshold of $p<0.05$ corrected for family-wise error (FWE). We then used a more conservative peak level threshold of $p<0.05$, corrected for multiple comparisons using the FWE correction method with a minimum cluster size of 19 voxels, based on the number of expected voxels per cluster ( $K$ $=18.563$ ).

Voxel-based correlation analysis was performed to determine the association between parameters (except the time spent in each block and brain volume in WKY rats). We used only significant voxels, which were detected by comparing the WKY and Wistar rats using the significant voxel map $(p<0.05$, 
cluster FWE-corrected) as a brain mask. An uncorrected $p<0.001$ with a minimum cluster size of 19 voxels was considered significant. Pearson's $r$ values were also calculated between parameters and mean grey matter voxel values of significant clusters.

\section{Declarations}

\section{Acknowledgements}

The authors thank Hikaru Morita for support in performing behavioural experiments. This study was supported by a Grant-in-Aid for Challenging Exploratory Research (17K18711) from the Ministry of Education, Culture, Sports, Science, and Technology of Japan (MEXT) (T.Y.) and a research grant from MSD K.K. (Tokyo, Japan) (T.Y.). This study was also supported in part by Grants-in-Aid for Scientific Research C (18K07712) from MEXT (N.O.).

\section{Conflict of Interest}

The authors declare no conflicts of interest.

\section{References}

1. World-Health-Organaization. Depression https://www.who.int/news-room/factsheets/detail/depression (2020).

2. Lim, G. Y. et al. Prevalence of Depression in the Community from 30 Countries between 1994 and 2014. Sci. Rep. 8, 2861, doi:10.1038/s41598-018-21243-x (2018).

3. Gerhard, D. M., Wohleb, E. S. \& Duman, R. S. Emerging treatment mechanisms for depression: focus on glutamate and synaptic plasticity. Drug Discov. Today 21, 454-464, doi:10.1016/j.drudis.2016.01.016 (2016).

4. Ashburner, J. \& Friston, K. J. Voxel-based morphometry-the methods. Neurolmage 11, 805-821, doi:10.1006/nimg.2000.0582 (2000).

5. Ashburner, J. Computational anatomy with the SPM software. Magn. Reson. Imaging 27, 1163-1174, doi:10.1016/j.mri.2009.01.006 (2009).

6. Du, M. Y. et al. Voxelwise meta-analysis of gray matter reduction in major depressive disorder. Prog. Neuropsychopharmacol. Biol. Psychiatry 36, 11-16, doi:10.1016/j.pnpbp.2011.09.014 (2012).

7. Bora, E., Fornito, A., Pantelis, C. \& Yucel, M. Gray matter abnormalities in Major Depressive Disorder: a meta-analysis of voxel based morphometry studies. J. Affect. Disord. 138, 9-18, doi:10.1016/j.jad.2011.03.049 (2012).

8. Lai, C. H. Gray matter volume in major depressive disorder: a meta-analysis of voxel-based morphometry studies. Psychiatry Res. 211, 37-46, doi:10.1016/j.pscychresns.2012.06.006 (2013).

9. Zhao, Y. J. et al. Brain grey matter abnormalities in medication-free patients with major depressive disorder: a meta-analysis. Psychol. Med. 44, 2927-2937, doi:10.1017/S0033291714000518 (2014). 
10. Zhang, H. et al. Brain gray matter alterations in first episodes of depression: A meta-analysis of whole-brain studies. Neurosci Biobehav Rev 60, 43-50, doi:10.1016/j.neubiorev.2015.10.011 (2016).

11. Peng, W., Chen, Z., Yin, L., Jia, Z. \& Gong, Q. Essential brain structural alterations in major depressive disorder: A voxel-wise meta-analysis on first episode, medication-naive patients. J. Affect. Disord. 199, 114-123, doi:10.1016/j.jad.2016.04.001 (2016).

12. Arnone, D. et al. Computational meta-analysis of statistical parametric maps in major depression. Hum. Brain Mapp. 37, 1393-1404, doi:10.1002/hbm.23108 (2016).

13. Hirschfeld, R. M. Differential diagnosis of bipolar disorder and major depressive disorder. J. Affect. Disord. 169, S12-S16, doi:10.1016/s0165-0327(14)70004-7 (2014).

14. Vai, B. et al. Predicting differential diagnosis between bipolar and unipolar depression with multiple kernel learning on multimodal structural neuroimaging. Eur. Neuropsychopharmacol. 34, 28-38, doi:10.1016/j.euroneuro.2020.03.008 (2020).

15. Wise, T. et al. Common and distinct patterns of grey-matter volume alteration in major depression and bipolar disorder: evidence from voxel-based meta-analysis. Mol. Psychiatry 22, 1455-1463, doi:10.1038/mp.2016.72 (2017).

16. Aleksandrova, L. R., Wang, Y. T. \& Phillips, A. G. Evaluation of the Wistar-Kyoto rat model of depression and the role of synaptic plasticity in depression and antidepressant response. Neurosci Biobehav Rev 105, 1-23, doi:10.1016/j.neubiorev.2019.07.007 (2019).

17. Will, C. C., Aird, F. \& Redei, E. E. Selectively bred Wistar-Kyoto rats: an animal model of depression and hyper-responsiveness to antidepressants. Mol. Psychiatry 8, 925-932, doi:10.1038/sj.mp.4001345 (2003).

18. Nam, H., Clinton, S. M., Jackson, N. L. \& Kerman, I. A. Learned helplessness and social avoidance in the Wistar-Kyoto rat. Front. Behav. Neurosci. 8, 109, doi:10.3389/fnbeh.2014.00109 (2014).

19. Burke, N. N. et al. Sex differences and similarities in depressive- and anxiety-like behaviour in the Wistar-Kyoto rat. Physiol Behav 167, 28-34, doi:10.1016/j.physbeh.2016.08.031 (2016).

20. Millard, S. J., Lum, J. S., Fernandez, F., Weston-Green, K. \& Newell, K. A. Perinatal exposure to fluoxetine increases anxiety- and depressive-like behaviours and alters glutamatergic markers in the prefrontal cortex and hippocampus of male adolescent rats: A comparison between Sprague-Dawley rats and the Wistar-Kyoto rat model of depression. J Psychopharmaco/33, 230-243, doi:10.1177/0269881118822141 (2019).

21. Solberg, L. C., Olson, S. L., Turek, F. W. \& Redei, E. Altered hormone levels and circadian rhythm of activity in the WKY rat, a putative animal model of depression. Am J Physiol Regul Integr Comp Physio/ 281, R786-794, doi:10.1152/ajpregu.2001.281.3.R786 (2001).

22. Pardon, M. C. e. a. Stress reactivity of the brain noradrenergic system in three rat strains differing in their neuroendocrine and behavioral responses to stress: implications for susceptibility to stressrelated neuropsychiatric disorders. Neuroscience Vol. 115, pp. $229 \wedge 242$ (2002)

23. Bruzos-Cidon, C. et al. Altered neuronal activity and differential sensitivity to acute antidepressants of locus coeruleus and dorsal raphe nucleus in Wistar Kyoto rats: a comparative study with Sprague 
Dawley and Wistar rats. Eur. Neuropsychopharmacol. 24, 1112-1122, doi:10.1016/j.euroneuro.2014.02.007 (2014).

24. Vinod, K. Y. et al. Dysfunction in fatty acid amide hydrolase is associated with depressive-like behavior in Wistar Kyoto rats. PLoS One 7, e36743, doi:10.1371/journal.pone.0036743 (2012).

25. O'Mahony, C. M., Clarke, G., Gibney, S., Dinan, T. G. \& Cryan, J. F. Strain differences in the neurochemical response to chronic restraint stress in the rat: relevance to depression. Pharmacol Biochem Behav 97, 690-699, doi:10.1016/j.pbb.2010.11.012 (2011).

26. Millard, S. J., Weston-Green, K. \& Newell, K. A. The Wistar-Kyoto rat model of endogenous depression: A tool for exploring treatment resistance with an urgent need to focus on sex differences. Prog. Neuropsychopharmacol. Biol. Psychiatry 101, 109908, doi:10.1016/j.pnpbp.2020.109908 (2020).

27. Willner, P. \& Belzung, C. Treatment-resistant depression: are animal models of depression fit for purpose? Psychopharmacology (Berl.) 232, 3473-3495, doi:10.1007/s00213-015-4034-7 (2015).

28. Abbott, R. D., Sherwin, K., Klopf, H., Mattingly, H. J. \& Brogan, K. Efficacy of a Multimodal Online Lifestyle Intervention for Depressive Symptoms and Quality of Life in Individuals With a History of Major Depressive Disorder. Cureus 12, e9061, doi:10.7759/cureus.9061 (2020).

29. Van Hasselt, V. B., Null, J. A., Kempton, T. \& Bukstein, O. G. Social skills and depression in adolescent substance abusers. Addict. Behav. 18, 9-18, doi:10.1016/0306-4603(93)90004-s (1993).

30. Malkesman, O., Maayan, R., Weizman, A. \& Weller, A. Aggressive behavior and HPA axis hormones after social isolation in adult rats of two different genetic animal models for depression. Behav Brain Res 175, 408-414, doi:10.1016/j.bbr.2006.09.017 (2006).

31. Mileva, G. R. \& Bielajew, C. Environmental manipulation affects depressive-like behaviours in female Wistar-Kyoto rats. Behav Brain Res 293, 208-216, doi:10.1016/j.bbr.2015.07.035 (2015).

32. D'Souza, D. \& Sadananda, M. Estrous Cycle Phase-Dependent Changes in Anxiety- and DepressionLike Profiles in the Late Adolescent Wistar-Kyoto Rat. Ann Neurosci 24, 136-145, doi:10.1159/000477151 (2017).

33. Mileva, G. R., Rooke, J., Ismail, N. \& Bielajew, C. Corticosterone and immune cytokine characterization following environmental manipulation in female WKY rats. Behav Brain Res 316, 197-204, doi:10.1016/j.bbr.2016.09.004 (2017).

34. Shetty, R. A. \& Sadananda, M. Immediate and delayed anxiety- and depression-like profiles in the adolescent Wistar-Kyoto rat model of endogenous depression following postweaning social isolation. Behav Brain Res 320, 323-332, doi:10.1016/j.bbr.2016.12.030 (2017).

35. Suzuki, H. et al. Structural abnormality of the hippocampus associated with depressive symptoms in heart failure rats. Neurolmage 105, 84-92, doi:10.1016/j.neuroimage.2014.10.040 (2015).

36. Depping, M. S., Schmitgen, M. M., Kubera, K. M. \& Wolf, R. C. Cerebellar Contributions to Major Depression. Front Psychiatry 9, 634, doi:10.3389/fpsyt.2018.00634 (2018).

37. Van Overwalle, F. et al. Consensus Paper: Cerebellum and Social Cognition. Cerebellum, doi:10.1007/s12311-020-01155-1 (2020). 
38. Sacchetti, B., Scelfo, B. \& Strata, P. Cerebellum and emotional behavior. Neuroscience 162, 756-762, doi:10.1016/j.neuroscience.2009.01.064 (2009).

39. Depping, M. S. et al. Cerebellar volume change in response to electroconvulsive therapy in patients with major depression. Prog. Neuropsychopharmacol. Biol. Psychiatry 73, 31-35, doi:10.1016/j.pnpbp.2016.09.007 (2017).

40. Yucel, K. et al. Cerebellar vermis volume in major depressive disorder. Brain Struct Funct 218, 851858, doi:10.1007/s00429-012-0433-2 (2013).

41. Coffman, K. A., Dum, R. P. \& Strick, P. L. Cerebellar vermis is a target of projections from the motor areas in the cerebral cortex. Proc Natl Acad Sci U S A 108, 16068-16073, doi:10.1073/pnas.1107904108 (2011).

42. Adamaszek, M. et al. Consensus Paper: Cerebellum and Emotion. Cerebellum 16, 552-576, doi:10.1007/s12311-016-0815-8 (2017).

43. Fanselow, M. S. \& Dong, H. W. Are the dorsal and ventral hippocampus functionally distinct structures? Neuron 65, 7-19, doi:10.1016/j.neuron.2009.11.031 (2010).

44. Floriou-Servou, A. et al. Distinct Proteomic, Transcriptomic, and Epigenetic Stress Responses in Dorsal and Ventral Hippocampus. Biol. Psychiatry 84, 531-541, doi:10.1016/j.biopsych.2018.02.003 (2018).

45. Gold, P. W. \& Kadriu, B. A Major Role for the Lateral Habenula in Depressive Illness: Physiologic and Molecular Mechanisms. Front Psychiatry 10, 320, doi:10.3389/fpsyt.2019.00320 (2019).

46. Ranft, K. et al. Evidence for structural abnormalities of the human habenular complex in affective disorders but not in schizophrenia. Psychol. Med. 40, 557-567, doi:10.1017/S0033291709990821 (2010).

47. Lawson, R. P. et al. Disrupted habenula function in major depression. Mol. Psychiatry 22, 202-208, doi:10.1038/mp.2016.81 (2017).

48. Carlson, P. J. et al. Neural correlates of rapid antidepressant response to ketamine in treatmentresistant unipolar depression: a preliminary positron emission tomography study. Biol. Psychiatry 73, 1213-1221, doi:10.1016/j.biopsych.2013.02.008 (2013).

49. Sherwin, E., Lennon, A. \& Harkin, A. Regional Specific Modulation of Stress-Induced Neuronal Activation Associated with the PSD95/NOS Interaction Inhibitor ZL006 in the Wistar Kyoto Rat. Int. J. Neuropsychopharmacol. 20, 833-843, doi:10.1093/ijnp/pyx053 (2017).

50. Blanchard, D. C., Blanchard, R. J., Takahashi, L. K. \& Takahashi, T. Septal lesions and aggressive behavior. Behav. Biol. 21, 157-161, doi:10.1016/s0091-6773(77)92407-5 (1977).

51. Albert, D. J. \& Chew, G. L. The septal forebrain and the inhibitory modulation of attack and defense in the rat. A review. Behav Neural Bio/30, 357-388, doi:10.1016/s0163-1047(80)91247-9 (1980).

52. Lorenzetti, V., Allen, N. B., Fornito, A. \& Yucel, M. Structural brain abnormalities in major depressive disorder: a selective review of recent MRI studies. J. Affect. Disord. 117, 1-17, doi:10.1016/j.jad.2008.11.021 (2009). 
53. Malykhin, N. V., Carter, R., Hegadoren, K. M., Seres, P. \& Coupland, N. J. Fronto-limbic volumetric changes in major depressive disorder. J. Affect. Disord. 136, 1104-1113, doi:10.1016/j.jad.2011.10.038 (2012).

54. Hickie, I. B. et al. Serotonin transporter gene status predicts caudate nucleus but not amygdala or hippocampal volumes in older persons with major depression. J. Affect. Disord. 98, 137-142, doi:10.1016/j.jad.2006.07.010 (2007).

55. Kronenberg, G. et al. Reduced amygdala volume in newly admitted psychiatric in-patients with unipolar major depression. J. Psychiatr. Res. 43, 1112-1117, doi:10.1016/j.jpsychires.2009.03.007 (2009).

56. Ferri, J. et al. Blunted amygdala activity is associated with depression severity in treatment-resistant depression. Cogn. Affect. Behav. Neurosci. 17, 1221-1231, doi:10.3758/s13415-017-0544-6 (2017).

57. Liu, C. H. et al. Voxel-based morphometry study of the insular cortex in female patients with current and remitted depression. Neuroscience 262, 190-199, doi:10.1016/j.neuroscience.2013.12.058 (2014).

58. Goodkind, M. et al. Identification of a common neurobiological substrate for mental illness. JAMA Psychiatry 72, 305-315, doi:10.1001/jamapsychiatry.2014.2206 (2015).

59. Consortium, C.-D. G. o. t. P. G. Identification of risk loci with shared effects on five major psychiatric disorders: a genome-wide analysis. The Lancet 381, 1371-1379, doi:10.1016/s0140-6736(12)621291 (2013).

60. Webb, C. A., Weber, M., Mundy, E. A. \& Killgore, W. D. Reduced gray matter volume in the anterior cingulate, orbitofrontal cortex and thalamus as a function of mild depressive symptoms: a voxelbased morphometric analysis. Psychol. Med. 44, 2833-2843, doi:10.1017/S0033291714000348 (2014).

61. Papp, M., Gruca, P., Faron-Gorecka, A., Kusmider, M. \& Willner, P. Genomic Screening of Wistar and Wistar-Kyoto Rats Exposed to Chronic Mild Stress and Deep Brain Stimulation of Prefrontal Cortex. Neuroscience 423, 66-75, doi:10.1016/j.neuroscience.2019.10.015 (2019).

62. Sturman, O., Germain, P. L. \& Bohacek, J. Exploratory rearing: a context- and stress-sensitive behavior recorded in the open-field test. Stress 21, 443-452, doi:10.1080/10253890.2018.1438405 (2018).

63. Molendijk, M. L. \& de Kloet, E. R. Immobility in the forced swim test is adaptive and does not reflect depression. Psychoneuroendocrinology 62, 389-391, doi:10.1016/j.psyneuen.2015.08.028 (2015).

64. Guarnieri, L. O. et al. Pro-neurogenic effect of fluoxetine in the olfactory bulb is concomitant to improvements in social memory and depressive-like behavior of socially isolated mice. Trans/ Psychiatry 10, 33, doi:10.1038/s41398-020-0701-5 (2020).

65. Stranahan, A. M., Khalil, D. \& Gould, E. Social isolation delays the positive effects of running on adult neurogenesis. Nat Neurosci 9, 526-533, doi:10.1038/nn1668 (2006).

66. Leasure, J. L. \& Decker, L. Social isolation prevents exercise-induced proliferation of hippocampal progenitor cells in female rats. Hippocampus 19, 907-912, doi:10.1002/hipo.20563 (2009). 
67. Fischer, C. W., Liebenberg, N., Elfving, B., Lund, S. \& Wegener, G. Isolation-induced behavioural changes in a genetic animal model of depression. Behav Brain Res 230, 85-91, doi:10.1016/j.bbr.2012.01.050 (2012).

68. Cahill, L. S. et al. Preparation of fixed mouse brains for MRI. Neurolmage 60, 933-939, doi:10.1016/j.neuroimage.2012.01.100 (2012).

69. Scheenstra, A. E. H. et al. Automated Segmentation of in Vivo and Ex Vivo Mouse Brain Magnetic Resonance Images. Mol. Imaging 8, doi:10.2310/7290.2009.00004 (2009).

70. Holmes, H. E. et al. Comparison of In Vivo and Ex Vivo MRI for the Detection of Structural Abnormalities in a Mouse Model of Tauopathy. Front. Neuroinform. 11, 20, doi:10.3389/fninf.2017.00020 (2017).

71. Kilkenny, C. et al. Animal research: reporting in vivo experiments: the ARRIVE guidelines. $\mathrm{Br} J$ Pharmacol 160, 1577-1579, doi:10.1111/j.1476-5381.2010.00872.x (2010).

72. Yoshii, T. et al. Brain atrophy in the visual cortex and thalamus induced by severe stress in animal model. Sci. Rep. 7, 12731, doi:10.1038/s41598-017-12917-z (2017).

\section{Tables}

Due to technical limitations, table 1 and 2 PDF's are only available as a download in the Supplemental Files section.

\section{Figures}



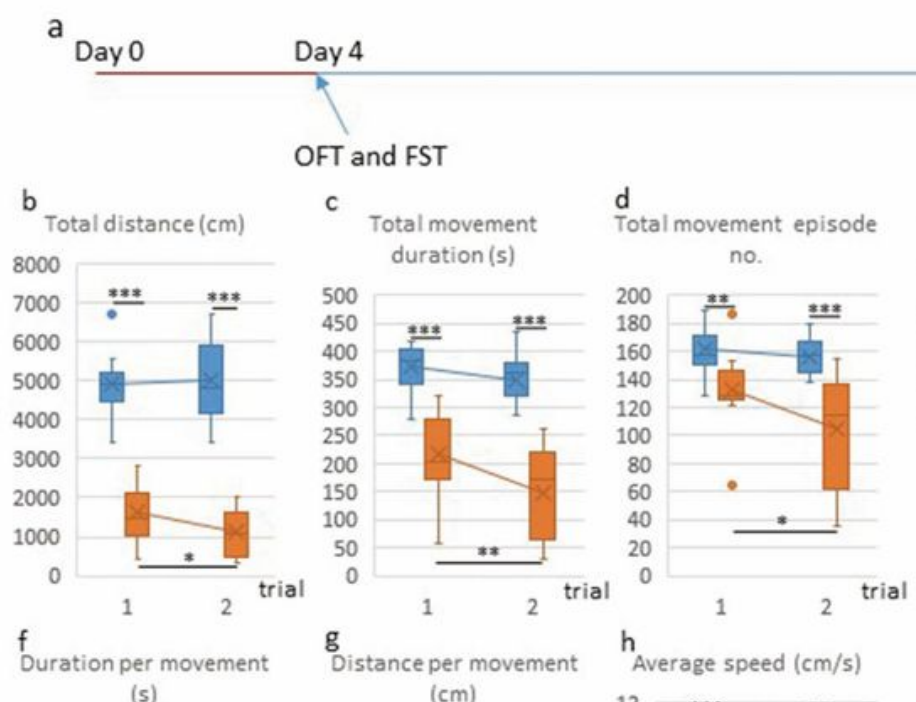

(s)
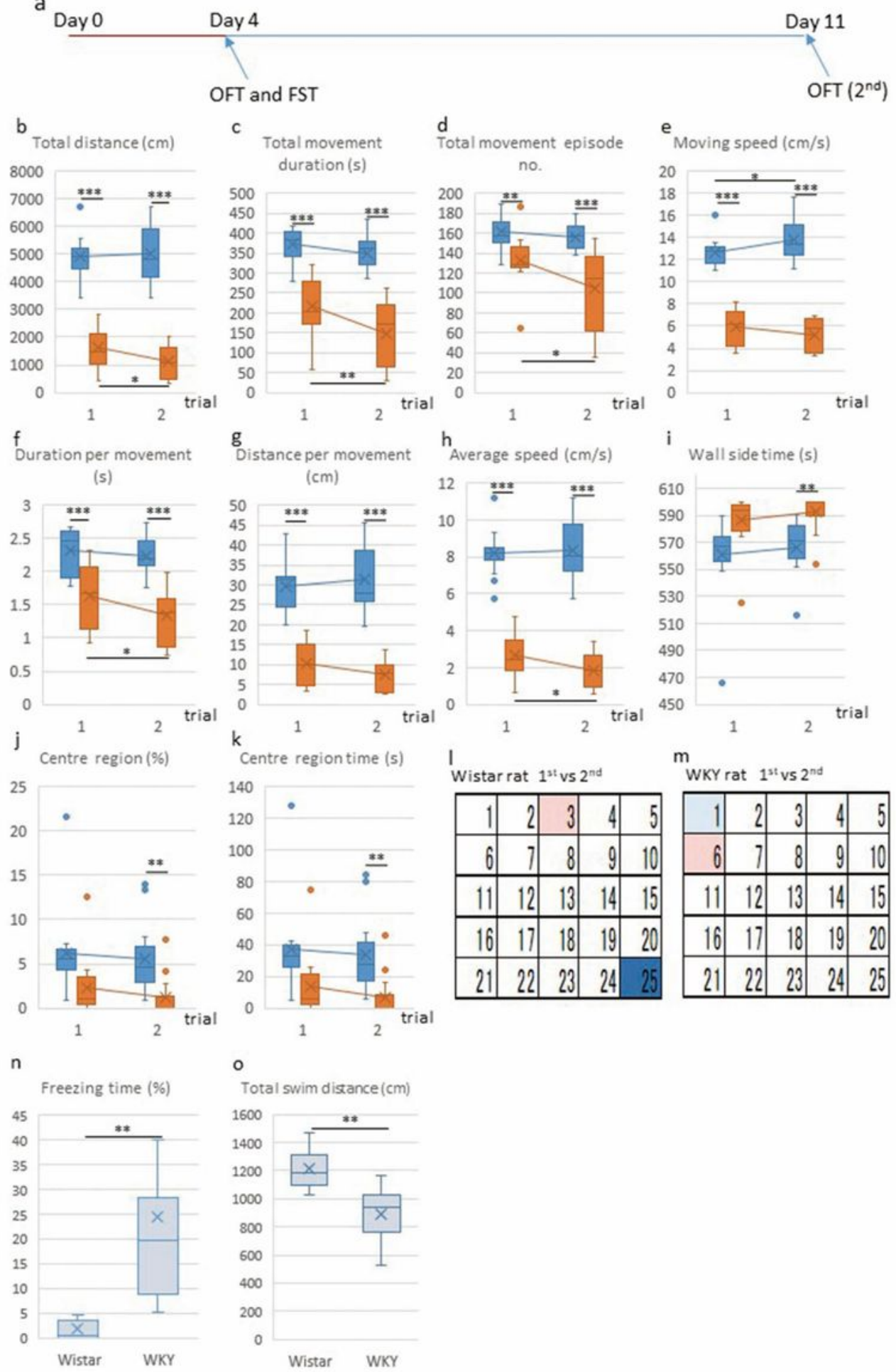

\section{Figure 1}

a) Schedule of behavioural experiments, including two open-field test (OFT) trials and one forced swim test (FST). The rats were subjected to an OFT and FST on Day 4 and sacrificed on Day 11, after the second OFT. b-k) Locomotion parameter measurements of Wistar $(n=13)$ and Wistar-Kyoto (WKY; $n=$ 13) rats from both OFT trials: b) total distance; c) total movement duration; d) total number of movement episodes; e) moving speed; f) duration per movement; g) distance per movement; $h$ ) average speed; i) 
wall-side time; j) percent of time spent in the centre region; and k) total amount of time spent in the centre region. $*: p<0.05 ; * \star: p<0.01, \star \star *: p<0.001$; analysis using repeated-measures general linear model (GLM) with Bonferroni correction with total intracranial volume (TIV) as a covariate. Blue box plots, Wistar rats; orange box plots, WKY rats. I, $\mathrm{m}$ ) Statistical heat maps for block stay durations between the two OFT trials overlaid on a schematic of the apparatus. I) Wistar rats, $n=13 . m$ ) WKY rats, $n=13$. Rats started to explore the open field from block 1 . Red shading indicates that block time during the second trial was less than the first trial; tint indicates statistical significance (light red: $p<0.05$; repeatedmeasures GLM with Bonferroni correction and TIV as a covariate). Blue shading indicates that time spent in the block during the first trial was less than the second trial; tint indicates statistical significance (light blue: $p<0.05$; dark blue: $p<0.001)$. $n$ ) Freezing time, defined as the percent of time spent immobile during the FST, was significantly higher in WKY rats $(n=13)$ than in Wistar rats $(n=11 ; t=-4.297 ; p<$ 0.01 ; two-tailed Welch's t-test with TIV as covariate). o) Total movement distance (cm) as a measure of locomotor activity during the FST was significantly shorter in WKY rats $(n=13)$ than in Wistar rats (Wistar: $\mathrm{n}=11 ; \mathrm{t}=4.404 ; \mathrm{p}<0.01$; two-tailed Welch's $\mathrm{t}$-test with TIV as covariate). 
a

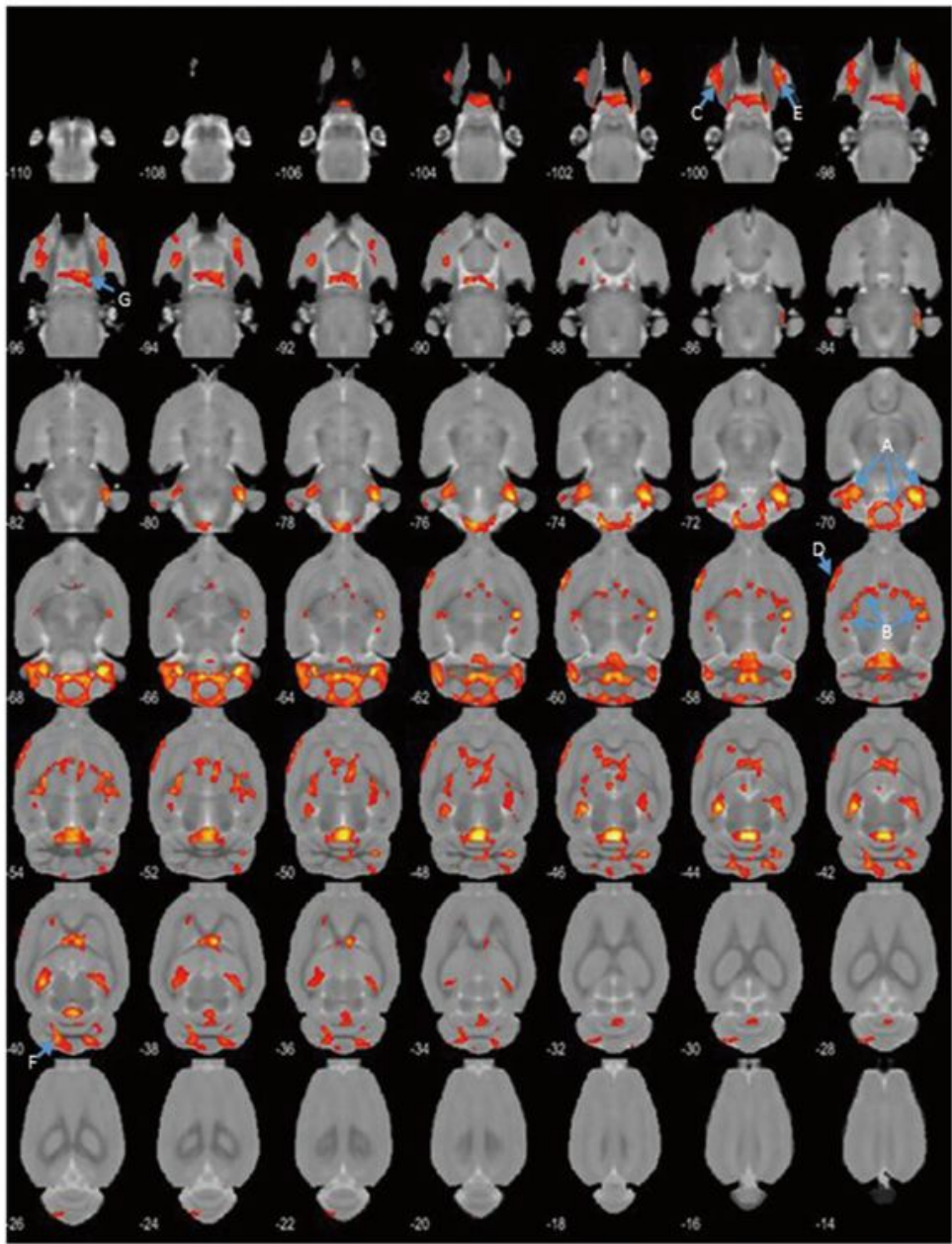

b

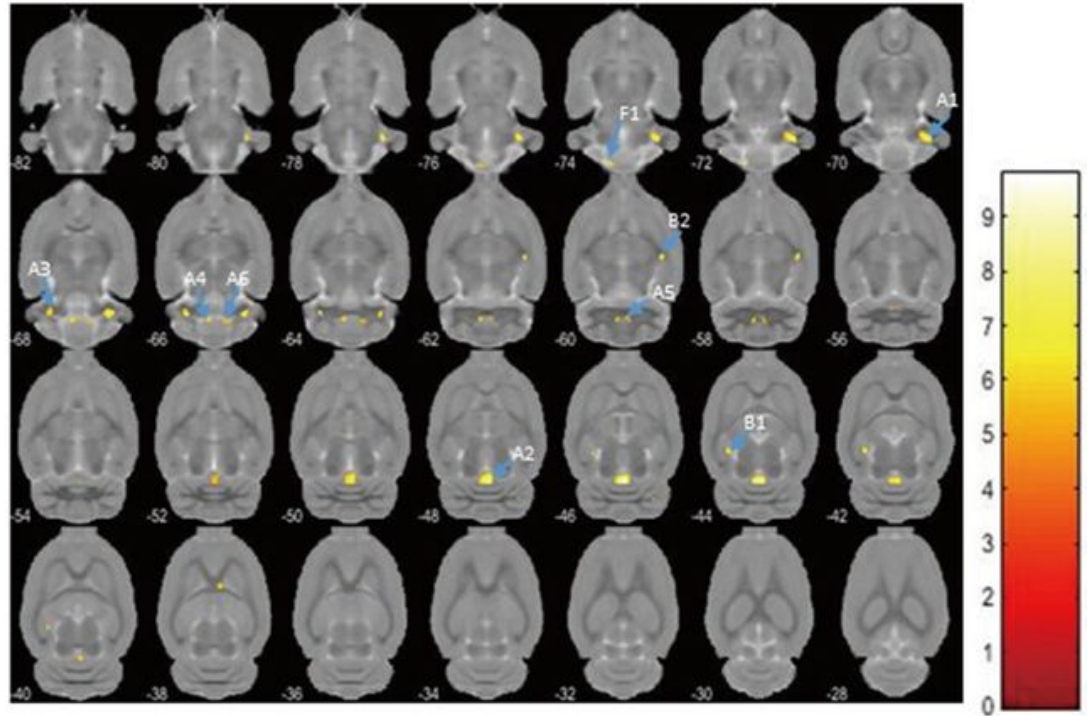

Figure 2

a) Axial brain slices showing strain differences resulting insignificant atrophy of part of the cerebellum (clusters $\mathrm{A}$ and $\mathrm{F}$ ), the bilateral hippocampus to the septum (cluster B), bilateral amygdala (clusters $\mathrm{C}$ and E), insular cortex (cluster D), and pituitary gland (cluster G). Height level, $p<0.001$; cluster family-wise error (FWE) corrected, $p<0.05$. b) Axial brain slices showing atrophy under the stricter threshold $(p<0.05$; FWE-corrected; minimum voxel size: 19). With this threshold, atrophic clusters were present in the 
cerebellar nuclei (A1 and A3-6), cerebellar vermis (A2 and F1), and parahippocampus (B1-3). The letters used are aligned with the cluster identification labels from the moderate threshold results. Colour bar units represent t-scores.

Negative correlation

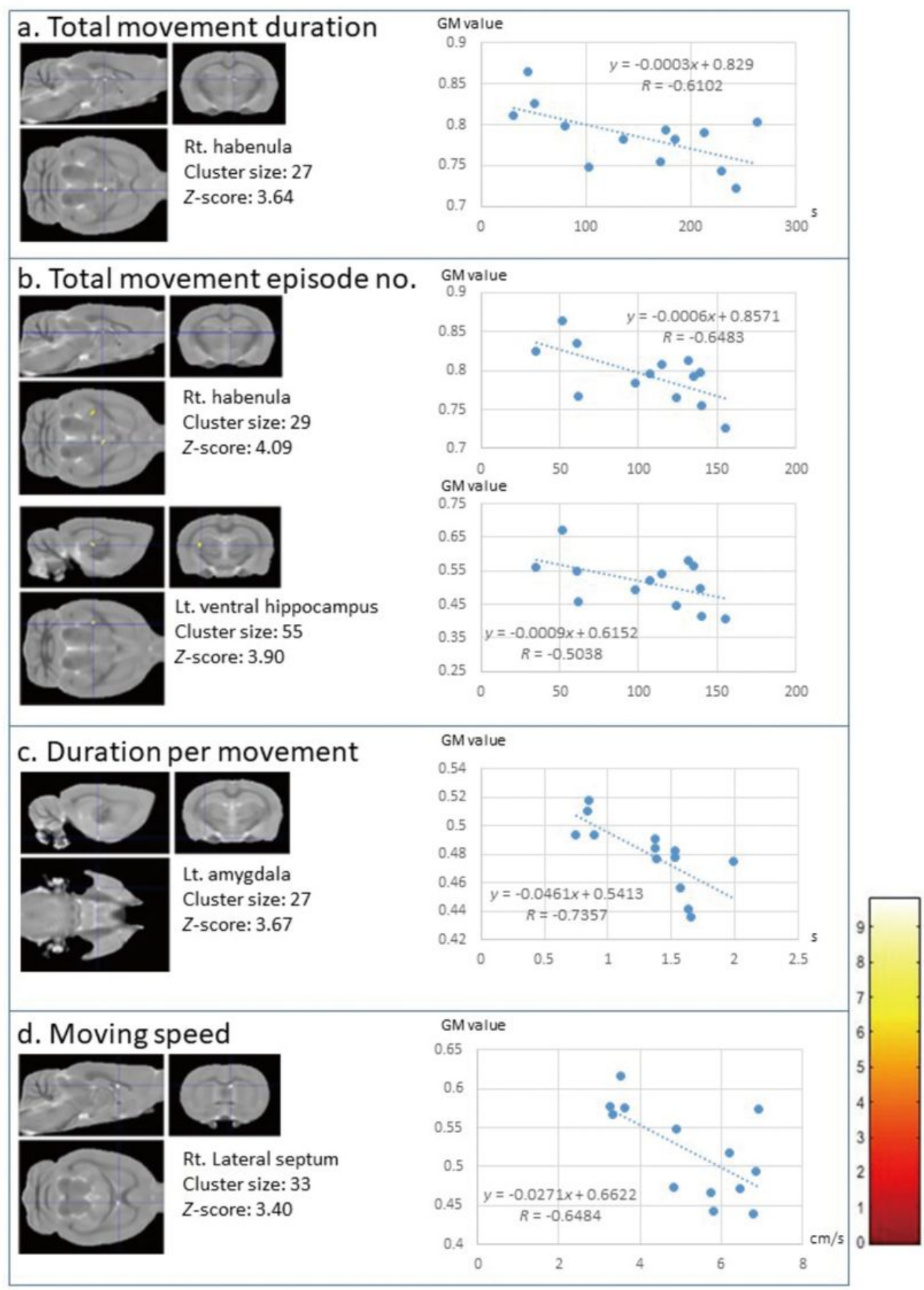

Figure 3

Clusters with negative correlations in Wistar-Kyoto (WKY) rats $(n=13)$. A threshold level of $p<0.001$, uncorrected for multiple comparisons, and a minimum of 19 voxels in a cluster were applied. a) Total 
movement duration. b) Total number of movement episodes. c) Duration per movement. d) moving speed. The negatively correlated clusters were in the right habenula, left ventral hippocampus, left amygdala, and right lateral septum. The adjacent graphs show the correlation between a given behaviour and the grey matter value inside the clusters detected by the voxel-based correlation analysis. Colour bar units represent t-scores.

\section{Positive correlation}

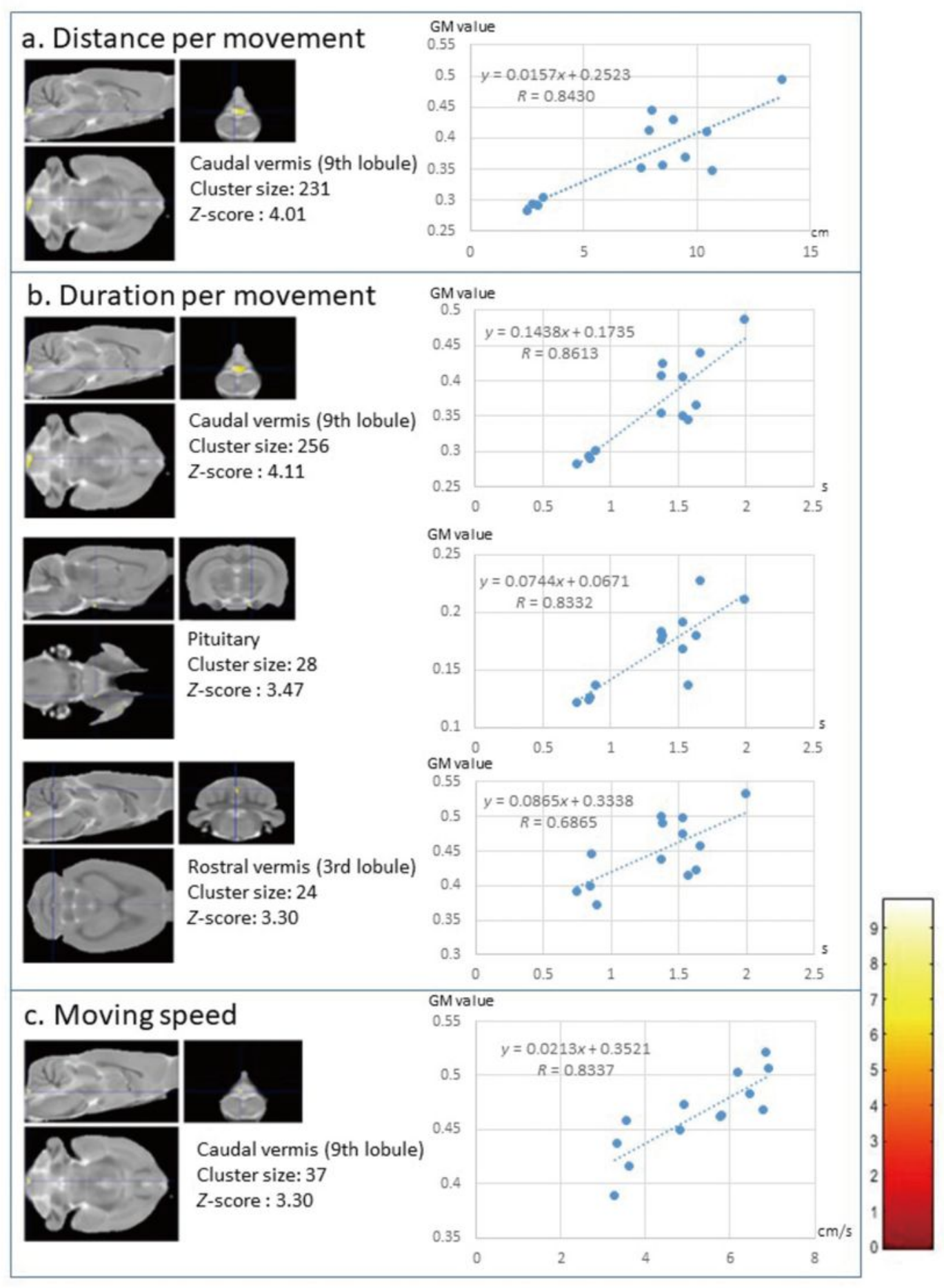

Figure 4 
Clusters with positive correlations in Wistar-Kyoto (WKY) rats $(n=13)$. A threshold level of $p<0.001$, uncorrected for multiple comparisons, and a minimum of 19 voxels in a cluster were applied. a) distance per movement. b) duration per movement. c) moving speed. The positively correlated clusters were located in the caudal vermis, rostral vermis, and pituitary gland. The adjacent graphs show the correlation between a given behaviour and the grey matter value inside the clusters detected by the voxel-based correlation analysis. Colour bar units represent t-scores.

\section{Supplementary Files}

This is a list of supplementary files associated with this preprint. Click to download.

- Supptable120210430.pdf

- Supptable220210430.pdf

- Supplementaryfigurelegend20210430.docx

- suppfig20210430.pdf

- table120210615.pdf

- table220210615.pdf 